\title{
Reply to 'UK set to reverse stance on research with chimeras'
}

To the editor:

I was very surprised that the news article on chimera research in the UK in your August issue ${ }^{1}$ contained a number of misuses of the terms 'hybrid' and 'chimera' and failed to distinguish between these two entirely distinct entities. Indeed, in places the article conflated the two terms, which is not helpful in informing the debate about these areas of research. There has been a lot of unnecessary and confusing controversy surrounding chimera research, much of which is routine and unremarkable, and it should be the role of journals such as Nature Medicine to reduce the confusion rather than to exacerbate it.

A hybrid is an organism whose cells all contain genetic material from two different species. Examples would be mules and many vegetables and garden flowers, which are generated by crosses between two species. 'Hybrid vigor' is frequently an advantage, and no one seems to object to larger vegetables or to more colorful or disease-resistant plants. Many hybrids, such as mules, are sterile, and hybrids can usually be made only between relatively closely related parents. No reputable scientist is suggesting making any sort of human hybrid, even if it were possible, which it probably is not.

In contrast, a chimera contains cells from two different organisms; there is no mingling of their genomes. Examples would include any individual who has received an organ transplant from another person, such as a transplant of the bone marrow, kidney or heart, as well as the chimeric mice that are generated in the course of gene knockout studies. These examples are intraspecies chimeras and are not controversial.

The controversies arise when people consider interspecies chimeras. Even in this case, the prospect of transplanting organs from pigs into humans seems acceptable to many people. The chimera research that has been the subject of debate involves various proposed experiments in which human embryonic stem cells or cells derived from them are introduced into other species, most commonly into mice. The transfer of human cells into other species is common-transplantation of human tumor cells into mice is routine in cancer research, for instance. When the time comes to test the efficacy of any human stem cells or their progeny cells in treating diseases, preclinical tests in mice (that is, chimera experiments) will be essential and will be required by the regulatory agencies.

There are some valid concerns about making interspecies chimeras with pluripotent stem cells_ such as the potential to affect neural development in the chimera-and those concerns need to be addressed. But many of the chimera experiments that will be a part of the development of stem-cell therapies are free of any such concerns.

Finally, the proposed experiments that have triggered the most recent furor in the UK involve the generation of something that is neither a hybrid nor a chimera. The researchers propose to insert a human somatic cell nucleus into the cytoplasm of the enucleated egg of an animal such as a cow or a rabbit. Such a construct, called a 'cybrid', has the nuclear genes of one organism combined with the cytoplasm of another.

The reason for doing this is to generate human pluripotent stem cell lines without using any human eggs, embryos or other reproductive material. This may or may not work, but no chimeric or hybrid animal-indeed no animal at all-would result.

So, why did these proposed experiments arouse so much controversy? In large part, this was caused by confusion about the nature of cybrids, hybrids and chimeras. In response to proposals to generate cybrids, the UK government initially proposed to ban research on all hybrid embryos, which was not even under consideration. Hybrids were then confused with chimeras, as in the first sentence-and elsewhere-in your article, and the popular press ran articles about minotaurs and other mythical beasts, further confusing the issues to be resolved. It is regrettable that a scientific journal such as Nature Medicine should add to this confusion. The distinctions between cybrids, hybrids and chimeras are unambiguous and should be kept clear.

\section{Richard Hynes}

Investigator, Howard Hughes Medical Institute, and Daniel K. Ludwig Professor for Cancer Research, Massachusetts Institute of Technology, Cambridge, Massachusetts 02139-4307, USA. email:rohynes@mit.edu

1. Hopkin, M. Nat. Med. 13, 890-891 (2007). 